\title{
Continuous subcutaneous apomorphine monotherapy in Parkinson's disease
}

\author{
Ewa Papuć1,A-D,F, Olga Trzciniecka, ${ }^{1, B}$, Konrad Rejdak ${ }^{1, A, E}$ \\ ${ }^{1}$ Department of Neurology, Medical University, Lublin, Poland \\ A - Research concept and design, B - Collection and/or assembly of data, C - Data analysis and interpretation, \\ $D$ - Writing the article, E - Critical revision of the article, F - Final approval of article
}

Papuć E, Trzciniecka O, Rejdak K. Continuous subcutaneous apomorphine monotherapy in Parkinson's disease. Ann Agric Environ Med. 2019; 26(1): 133-137. doi: 10.26444/aaem/99699

\begin{abstract}
Introduction and objective. Continuous subcutaneous apomorphine (APO) treatment is one of the 3 therapeutic options for advanced Parkinson's disease (PD), in addition to deep brain stimulation (DBS) and intrajejunal levodopa. Data from previously performed studies show that few PD patients can achieve APO infusion as monotherapy. The current pilot study presents the authors' experience in achieving APO monotherapy.

Materials and method. During the last 2 years, 9 patients with APO were treated in the Department of Neurology of the Medical University of Lublin; each patient was offered a 5-day duration APO treatment as monotherapy. The main indication for the APO therapy was advanced PD with motor fluctuations and the patient's non-agreement for DBS therapy. Mean age of treated patients - 65.11 years, mean disease duration - 7.67 years, mean Hoehn-Yahr - 2.67, mean L-dopa equivalent before APO treatment - $1751.11 \mathrm{mg}$, mean daily dose of apomorphine as monotherapy - $106.11 \pm 14.09 \mathrm{mg}$.

Results. All treated patients managed to achieve APO monotherapy. A statistically significant reduction was found in the duration of the 'off' states in the observed PD patients on APO monotherapy $(p<0.05)$. No significant improvement was observed in the III motor score of the UPDRS on APO treatment, compared to optimized oral therapy used before APO treatment.

Conclusions. APO monotherapy can be achieved in advanced PD, and seems to be a good therapeutic option for this group of patients, especially in that it allows a significant reduction in the off-time which significantly simplifies the drug regime. Nevertheless, hospital admission with experienced neurologist supervision is recommended when establishing a PD patient's APO monotherapy.
\end{abstract}

\section{Key words}

Parkinson's disease, apomorphine, continuous subcutaneous infusion, monotherapy, apomorphine pump

\section{INTRODUCTION}

Continuous subcutaneous apomorphine (APO) infusion is one of the 3 therapeutic options in advanced Parkinson's disease (PD), in addition to intrajejunal levodopa infusion and deep brain stimulation (DBS) [1]. After a few years of oral therapy, the majority of PD patients experience motor and non-motor complications, as well as levodopa induced dyskinesias. APO is mainly indicated for patients with motor fluctuations and peak dose dyskinesia; additionally, the drug also has very good efficacy in the reduction of nonmotor fluctuations and can also be used to reverse mainly predictable 'off' periods. The subcutaneous form of APO delivery improves drug bio-availability and allows faster onset of action due to the avoidance of gastrointestinal transit time.

Apomorphine is a D1 and D2 receptor dopamine agonist, and given subcutaneously should have at least the same efficacy as oral levodopa [2]. Apomorphine has been used over many centuries as an emetic, sedative, or even as an anticonvulsive drug [3], but the first suggestions for its use in Parkinson's disease were indicated by Weil in 1844 . Initially the wide use of apomorphine was limited, mainly because of its side-effects - nausea and vomiting, which

Address for correspondence: Ewa Papuć, Department of Neurology, Medical University, Jaczewskiego 8, 20-954 Lublin

e-mail: ewapap@yahoo.pl

Received: 16.07.2018; accepted: 11.11.2018; first published: 05.12.2018 reflected its dopaminergic activity. Only the introduction in Europe of peripherally acting dopamine antagonists, such as domperidone, and trimethobenzamide in the USA, allowed its wider use in the late 1990s [4]. Apomorphine's ability to reduce both motor and non-motor symptoms, as well as its side-effects, have been evaluated in many studies $[5,6,7,8]$.

Intrajejunal levodopa infusion and deep brain stimulation are also beneficial in advanced PD; nevertheless, some patients may have contraindications for invasive methods, and the personal preference of the patient should also be taken into consideration. Some studies have compared apomorphine with DBS $[9,10]$ or with intrajejunal levodopa; none of these studies, however, were placebo controlled [11]. As for apomorphine, only one placebo controlled double blind study has been published recently, which may be the key reason for the lack of reimbursement for this drug in some countries and its limited availability [12]. Subcutaneous apomorphine infusion has just started to be reimbursed in Poland, but experience with this drug is lacking.

\section{OBJECTIVES}

Subcutaneous apomorphine treatment minimizes the risk of pulsatile dopaminergic stimulation, which is why the aim of this study was to switch the PD patients from optimal oral therapy to continuous subcutaneous treatment. Data from previously published studies revealed that it is difficult 
to achieve monotherapy in advanced PD subjects. In the studies published so far, data on the mean reduction of the L-dopa equivalent dose and rates of patients who achieved monotherapy with APO varied widely [7, 13, 14, 15]. For this reason, this study presents the authors' experience with subcutaneous apomorphine infusion given as monotherapy in advanced PD patients. Also presented are their experience with introducing APO therapy.

\section{MATERIALS AND METHOD}

Since 2009, another therapy for advanced Parkinson's disease, deep brain stimulation (DBS) has been in use. As this therapy has for many years been the only one reimbursed in Poland, it was considered as an option (DBS) in all PD patients in advanced stage with motor fluctuations, who despite optimized oral therapy, fulfilled the criteria of the CAPSIT protocol (Core Assessment Program for Surgical Interventional Therapies in Parkinson's Disease [16].

Over the past 2 years, 9 consecutive patients who fulfilled the criteria for DBS according to the CAPSIT protocol, but did not give their consent for DBS therapy, were offered the possibility to test a 5-day continuous subcutaneous apomorphine infusion as monotherapy. All patients met the Postuma criteria for PD [17] and all of them had severe motor fluctuations. This possibility was offered to the patients in the hope of imminent reimbursement for this drug, because at the time of designing this small pilot study the drug was not reimbursed in Poland. Contraindications for APO treatment were: 1) severe psychosis in the history, 2) impulse control disorder, 3) severe disabling dyskinesia, 4) dementia, 5) symptomatic orthostatic hypotension, and 6) cardiac arrhythmia.

All the patients had ECG performed before introducing APO and domperidone therapy in order to exclude prolonged QT duration, atrial fibrillation or premature ventricular contractions. Baseline blood morphology was also performed to exclude haemolytic anaemia.

The pump was installed during the patients' hospitalization. As the therapy was of 5-day duration, a quick switch was made from levodopa to apomorphine, but dopamine agonists were gradually withhold within $2-5$ days. In addition to continuous subcutaneous APO delivery, patients may require additional boluses of APO. The optimal bolus dosage of APO has to be determined on an individual patient basis; therefore, APO treatment was started by establishing the threshold dose, which is individual for each patient. For this, the 'off' phase in motor performance was provoked by withholding all antiparkinsonian drugs overnight (12 hours). Only dopamine agonist were withhold gradually, 2-5 days before provoking the 'off' phase in the patient. Baseline motor assessment was performed in the III motor part of the UPDRS in each patient before administering APO, but on optimized oral treatment [18]. It is also additionally recommended that each patient should be on the anti-emetic domperidone for at least 48-72 hours prior to initiation of therapy. As domperidone is not commonly available in Poland, it was not used in the current study as a routine prophylaxis treatment, but only in certain cases where nausea and vomiting occurred.

The threshold APO dose was established in each individual patient according to the following schedule: $1 \mathrm{mg}$ of APO solution injected subcutaneously, and the patient assessed after 30 minutes for motor response. If no or poor response was observed, a consecutive dose of $2 \mathrm{mg}$ apomorphine solution was given, on average 40 minutes after the first dose, and the patient observed and assessed again after 30 minutes. If again no satisfactory response was obtained, 40 minutes after the $2^{\text {nd }}$ dose, a $3^{\text {rd }}$ dose was given, and again the motor response assessed 30 minutes later. The dosage was increased every 40 minutes by $1 \mathrm{mg}$, until unequivocal motor response was observed in the patient. The lowest dose which produced unequivocal motor response in the patient was determined as threshold dose. The threshold dose for all the patients was 3-4 mg.

Following establishment of the threshold dose, continuous subcutaneous infusion was started using a portable syringe driven pump. The therapy was continued for 5 consecutive days, in 7 patients continuous infusion lasted 16-18 hours, with an overnight break lasting 6-8 hours, depending on the tolerance of the patient. In addition, 2 patients required oral long-lasting levodopa treatment given once per night. Two patients required continuous nocturnal subcutaneous apomorphine infusion due to 'off' states experienced during the night which poorly responded to oral control release levodopa treatment. In these patients, the infusion was given for 22 hours. The majority of patients (8 of 9) required additional boluses during the continuous APO infusion, on average 1-2 boluses per day, due to the 'off' states. The patients' motor situation was assessed using UPDRS part III in the 'on' and 'off' before the APO treatment and in the 'on' state on APO treatment, because patients experienced almost no 'off' periods.

Improvement in the patients' motor performance and quality of life on APO monotherapy was performed using the Clinical Global Impressions-Improvement (GCI-I) scale by a neurologist experienced in movement disorders [19]. The patients were assessed on the $5^{\text {th }}$ day of APO monotherapy.

Statistical analysis. Data were presented as means with standard deviation. Comparison of the number of hours before APO and on APO treatment was performed using the Wilcoxon paired samples test. $P$ value $<0.05$ was considered statistically significant (two-sided). Statistical calculations were performed using Statistica 9.1 Programme.

\section{RESULTS}

Group characteristics. There were 9 PD patients in the studied group, all of whom managed to obtain monotherapy. Demographic and clinical data regarding the patients before APO treatment are presented in Table 1. Clinical data regarding apomorphine treatment and motor performance on APO are presented in Table 2.

The APO infusion was continued for 5 days in all investigated patients, with good tolerance.

Apomorphine therapy tolerance and side-effects. For 2 out of 9 patients, APO therapy was very well tolerated and not connected with any side-effects. 2 other patients had very poor tolerance of overnight break and required continuation of therapy also during the night (continuous 22 hour infusion). 2 other patients required an additional prescription of long-lasting levodopa oral treatment once per night. Although some side-effects were observed, they 
Table 1. Baseline demographic and clinical characteristics of PD patients before apomorphine treatment

\begin{tabular}{lcc}
\hline & Mean \pm SD & Range \\
\hline Age (years) & $65.11 \pm 8.51$ & $51-75$ \\
\hline Disease duration (years) & $7.67 \pm 1.32$ & $6-10$ \\
\hline $\begin{array}{l}\text { Levodopa equivalent before apomorphine } \\
\text { treatment (mg/24h) }\end{array}$ & $1751.11 \pm 199.59$ & $1550-2200$ \\
\hline $\begin{array}{l}\text { Pure levodopa dose before apomorphine } \\
\text { treatment (mg/24h) }\end{array}$ & $744.44 \pm 325.43$ & $300-1500$ \\
\hline $\begin{array}{l}\text { Hoehn-Yahr stage before apomorphine } \\
\text { treatment }\end{array}$ & $2.67 \pm 0.50$ & $2-3$ \\
\hline $\begin{array}{l}\text { Hoehn-Yahr stage on apomorphine treatment } \\
\begin{array}{l}\text { UPDRS in the ON state before apomorphine } \\
\text { treatment }\end{array}\end{array}$ & $46.00 \pm 6.44$ & $42-66$ \\
\hline $\begin{array}{l}\text { UPDRS in the OFF state before apomorphine } \\
\text { treatment }\end{array}$ & $67.44 \pm 6.97$ & $54-79$ \\
\hline $\begin{array}{l}\text { UPDRS in the ON state on apomorphine } \\
\text { treatment }\end{array}$ & $43.22 \pm 12.12$ & $28-66$ \\
\hline
\end{tabular}

UPDRS - unified Parkinson's disease rating scale; SD - standard deviation.

Table 2. Clinical characteristics of PD patients receiving apomorphine treatment

\begin{tabular}{|c|c|c|}
\hline Threshold dose of apomorphine (mg) & $3.22 \pm 0.44$ & $3-4$ \\
\hline Mean daily dose of apomorphine (mg/24h) & $106.11 \pm 14.09$ & $90-125$ \\
\hline Number of boluses per day & $1.44 \pm 0.73$ & $0-2$ \\
\hline $\begin{array}{l}\text { Number in OFF hours before apomorphine treatment } \\
(\mathrm{h} / 24 \mathrm{~h})\end{array}$ & $5.22 \pm 2.86$ & $2-12$ \\
\hline $\begin{array}{l}\text { Number in OFF hours on apomorphine treatment } \\
\text { (h/24h) }\end{array}$ & $0.72 \pm 0.26$ & $0.5-1$ \\
\hline $\begin{array}{l}\text { Overall dose of apomorphine per 5-day treatment } \\
\text { (mg) }\end{array}$ & $521.67 \pm 70.00$ & $410-660$ \\
\hline Mean flow of apomorphine $(\mathrm{mg} / \mathrm{h})$ & $6.00 \pm 0.12$ & $5.2-6.5$ \\
\hline Overnight break in apomorphine treatment $(\mathrm{h} / 24 \mathrm{~h})$ & $6.78 \pm 0.67$ & $2-8$ \\
\hline Total daily hours on apomorphine & $17.66 \pm 1.73$ & $16-22$ \\
\hline UPDRS in the ON state on apomorphine treatment & $43.22 \pm 12.12$ & $28-66$ \\
\hline
\end{tabular}

UPDRS - unified Parkinson's disease rating scale; SD - standard deviation.

Table 3. Observed side-effects of apomorphine therapy in the investigated group of patients.

\begin{tabular}{lc}
\hline Side-effect & No. of patients \\
\hline Dizziness & $3 / 9$ \\
\hline Nausea & $5 / 9$ \\
\hline Vomiting & $4 / 9$ \\
\hline Visual hallucinations & $1 / 9$ \\
\hline
\end{tabular}

were mild and did not hinder continuation of APO therapy (Tab. 3).

No hypotension, dyskinesia, cardiac arrhythmia, skin rash, or other allergic type reactions were observed in any of the patients. Other side-effects, e.g. impulse control disorder, haemolytic anaemia or skin nodules were also not observed in the patients. However, such effects may occur during longer treatment.

Motor functionality. Mean UPDRS part III in the 'on' state did not differ significantly between the optimized oral

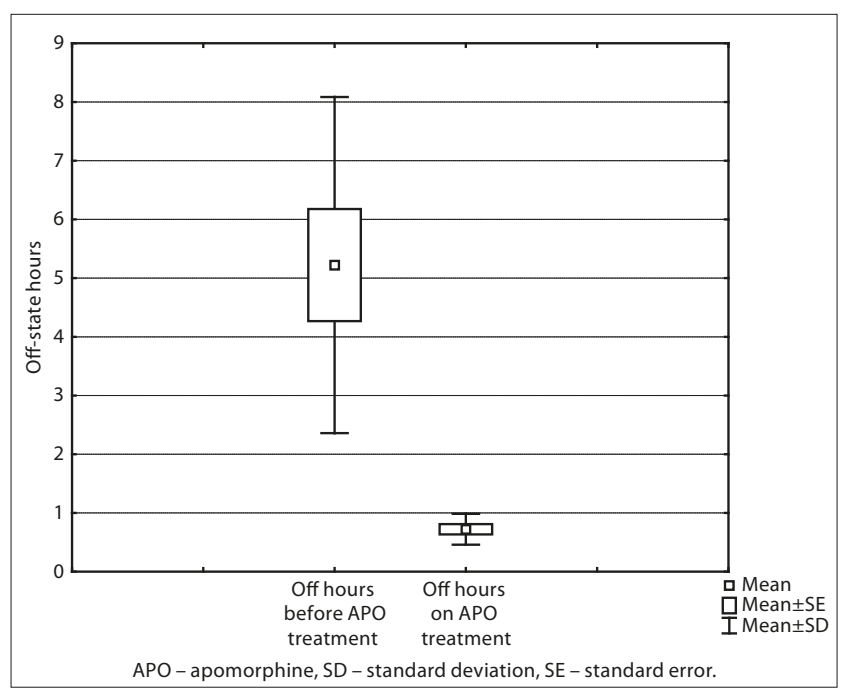

Figure 1. Difference in the 'off' hours before APO treatment and on APO treatment. Average reduction in length of 'off' states on APO treatment -4.5 hours ( $p=0.007$; $Z=2.66$ )

therapy (levodopa+dopamine agonist) and APO infusion (p>0.05) (Tab. 2). Nevertheless, a significant reduction was observed in the length of daily 'off' hours (5.22 $\pm 2.86-$ $0.72 \pm 0.26$ ); $\mathrm{p}<0.05$ ) (Fig. 1).

The motor function and the patient's quality of life, assessed by an experienced neurologist in CGI-I scale on the APO monotherapy, was assessed as very much improved in 5 of 9 patients (55\%), and as much improved in 4 of 9 patients (45\%). No patient was assessed as being worse or without any change

\section{DISCUSSION}

This small pilot study confirms that continuous subcutaneous apomorphine delivery given as monotherapy can be an effective and well tolerated therapeutic option for advanced PD. Apomorphine generally can be given in single injections in those PD patients who experience rapid relief in predictable and unpredictable 'off' periods, and also to PD patients with levodopa absorption or gastric emptying problems. The drug can also be administered in a continuous infusion to those PD patients who experience frequent 'off' episodes (more than 4-6 per day) [20]. Continuous apomorphine infusion given as monotherapy results in an almost continuous 'on' state, as confirmed by this study. During the presented APO monotherapy, only 2 patients experienced no 'off'” states, as the maximum overall 'off' time was one hour per day.

The current study confirms that APO monotherapy allowed good control of PD symptoms, with significant reduction of the length of the 'off' states. An average 4.5 hour reduction was observed in the 'off' state per day. The obtained results are similar to those obtained in the Manson's study, where an increase by 4.5 hours in the daily 'on' period was also observed [8]. This is longer than the time described by some other authors $[5,8,21]$. However, the limitation of the current study is the very short 5-day observational period and small number of PD patients included. Of course it cannot be excluded that an additional placebo effect would potentially influence such a good results, but would definitely not lead to the extension of the 'on' state by itself. Using oral or transdermal dopamine agonists, the 'on' time can be 
prolonged by about 2 hours per day [8]; thus. apomorphine allows even better control of motor fluctuations among PD patients. This happens partially because apomorphine differs from oral dopamine agonists: apomorphine interacts with both D1 and D2 receptor classes, and with all major types of dopamine receptors from D1 to D5, whereas oral dopamine agonists like ropinirole or pramipexol have limited interaction with different dopamine receptors [2]. This limited interaction with different dopamine receptors has important clinical consequences, as oral dopamine agonist never have such as good antiparkinsonian efficacy as levodopa or apomorphine [22]. The mode of continuous subcutaneous drug delivery also plays a key role in the beneficial effect on motor performance.

As for adjustement of apomorphine bolus dose, all patients in this study responded to a dose of 3-4 $\mathrm{mg}$ given s.c. The optimal dose should be adjusted on an individual basis, but if any patients present no response to the $7 \mathrm{mg}$ dose of APO, then they must be classified as non-responders and no further attempts should be made. There were no non-responding patients to APO in the current study.

All side-effects in the patients were transient and easy manageable. The most common were nausea and vomiting, which occurred in 5 out of 9 patients. Gastrointestinal side-effects are generally the most frequently described in the literature $[8,20,23]$, but they do not seem to be the serious or persistent [20]. Nausea and vomiting occur more frequently for acute apomorphine injections than for continuous infusion. What is more important, tolerance to these side-effects develops rapidly. As mentioned above, no domperidone was administered in the prophylaxis treatment as it is not available in Poland, and has to be imported from abroad. Only 2 patients required domperidone, and this was only for 2 consecutive days, as later nausea and vomiting resulted and domperidone was withheld. In the remaining patients, nausea and vomiting were transient, of mild intensity, occurred only at the beginning of the therapy, and resolved spontaneously.

It is worth noting, that all patients in this study were on apomorphine monotherapy, whereas in the literature data on APO monotherapy, the rates among advanced PD patients vary widely. The presented results are in accordance with results of the Manson's study performed in 2002, where the majority of patients (70\%) achieved APO monotherapy (45 of 64 patients) [7]. Contrary to these results, in a study by A. Sesar et al. in 2017, none of their 93 patients managed to achieve monotherapy [8]. Also, H. L. Tyne et al. [15] and R. Borgemeester et al [14] revealed very low rates of APO monotherapy in their studies. It should be highlighted here in the current study apomorphine monotherapy is defined as administering apomorphine as the only drug during the daytime, with a single dose of long- lasting levodopa given per night, as undertaken by some other authors $[7,8,13]$.

In the presented study, APO monotherapy was not be the goal itself, but rather a reduction in the daily 'off' states among the PD patients, as well as improvement in the patients' quality of life. Nevertheless, APO monotherapy may also allow avoidance of the side-effects of levodopa treatment, improvement in drug compliance, and make the patient less dependent on oral treatment.

Although aomorphine is usually recommended to be administered only during daytime, with an overnight break, if the control of parkinsonian symptoms during the night seems to be poor, it can be also given in a nocturnal infusion [21]. In the presented study, this treatment was administered to only to 2 patients, who, as mentioned above, had poor control of nocturnal parkinsonian symptoms. The therapy was well tolerated; nevertheless, with longer-lasting treatment local adverse events may occur to a more severe degree, especially skin nodules. It should be highlighted here that the infusion site should be changed every 12 hours. There is evidence from the literature that continuous 24-hour infusion may potentially lead to tolerance and an impaired response to apomorphine, although this phenomenon is reversible $[23,24]$.

It must be highlighted that it is very important to define the advanced stage of PD and to refer the patient timely to a suitable advanced PD therapy. Advanced PD occurs when optimally adjusted oral treatment does not provide good control of motor and non-motor symptoms. It is at this stage that the patient should be offered second line therapy, e.g. DBS, intrajejunal levodopa or continuous subcutaneous apomorphine infusion.

This study confirms that an excellent control of both motor symptoms may be achieved with APO monotherapy, with minimal adverse events and minimal invasiveness for the patient $[21,25]$. Also, according to the Expert Consensus Group, continuous APO therapy is less invasive than intrajejunal levodopa infusion or deep brain stimulation [20]. It is worth noting that sometimes it can be the only therapeutic option when other, invasive interventions like DBS or inrajejunal levodopa infusion, are not accepted by the patient. Unlike DBS, continuous apomorphine treatment is neither contraindicated in patients with advanced age, nor in those with mild cognitive impairment [20]. What is more important is that patients on apomorphine do not deteriorate with time in their neuropsychiatric performance, compared to DBS $[9,10,26]$.

In addition, the use of apomorphine pump as monotherapy can simplify the treatment in the advanced stage of PD as it can be a good alternative option when a patient is on a complex oral dosing regimen. Complex oral treatment in advanced PD can strongly influence adherence to therapy [27], and the use of apomorphine monotherapy definitely improves this adherence.

Apomorphine seems to be an interesting drug, with positive influence not only on motor but also non-motor symptoms. The current study did not assess this non-motor influence, although data from many studies suggest that it can have beneficial effects, such as for some aspects of sleep (reduce restless legs syndrome, nocturnal or early morning off episodes). APO may also decrease pain related to the 'off' states, may improve mood and reduce urinary incontinence $[11,28,29]$. It is also interesting that data from neuropathological animal studies suggest that it can be a potential modifier of amyloid deposition, which can be of importance as the majority of $\mathrm{PD}$ patients develop cognitive impairment at certain stages of the disease [30].

\section{CONCLUSIONS}

The results of this small pilot study show that continuous subcutaneous apomorphine monotherapy treatment seems to be a good therapeutic option for advanced PD. The major benefit of this therapy is that it allows significant reduction in 
the patients' daily 'off' states. The APO therapy is well tolerated, as potential side-effects are easily manageable, usually by dose adjustment. Hospital admission is recommended under the supervision of an experienced neurologist when starting the therapy, and thereafter establishing a patient's therapeutic regime.

\section{REFERENCES}

1. Antonini A, Moro E, Godeiro C, Reichmann H. Medical and surgical management of advanced Parkinson's disease. Mov Disord. Online Early https:/onlinelibrary.wiley.com/doi/abs/10.1002/mds.27340.

2. Jenner P, Katzenschlager R. Apomorphine - pharmacological properties and clinical trials in Parkinson's disease. Parkinsonism Relat Disord. 2016; 33(Suppl1): 13-21.

3. Lees AJ. Dopamine agonists in Parkinson's disease: a look at apomorphine. Fundam Clin Pharmacol. 1993; 7(3-4): 121-128.

4. Colosimo C, Merello M, Albanese A. Clinical usefulness of apomorphine in movement disorders. Clin Neuropharmacol. 1994; 17(3): 243-259.

5. Obeso JA, Grandas F, Vaamonde J, Rosario Luguin M, MartínezLage JM. Apomorphine infusion for motor fluctuations in Parkinson's disease. Lancet. 1987; 1(8546): 1376-1377.

6. Stocchi F, Vacca L, De Pandis MF, Barbato L, Valente M, Ruggieri S. Subcutaneous continuous apomorphine infusion in fluctuating patients with Parkinson's disease: long-term results. Neurol Sci. 2001; 22(1): 93-94.

7. Manson AJ, Turner K, Lees AJ. Apomorphine monotherapy in the treatment of refractory motor complications of Parkinson's disease: long-term follow-up study of 64 patients. Mov Disord. 2002; 17(6): 1235-1241.

8. Sesar Á, Fernández-Pajarín G, Ares B, Rivas MT, Castro A. Continuous subcutaneous apomorphine infusion in advanced Parkinson's disease: 10-year experience with 230 patients. J Neurol. 2017; 264(5): 946-954

9. Antonini A, Isaias IU, Rodolfi G, Landi A, Natuzzi F, Siri C, et al. A 5 -year prospective assessment of advanced Parkinson disease patients treated with subcutaneous apomorphine infusion or deep brain stimulation. J Neurol. 2011; 258(4): 579-585.

10. De Gaspari D, Siri C, Landi A, Cilia R, Bonetti A, Natuzzi F, et al. Clinical and neuropsychological follow up at 12 months in patient with complicated Parkinson's disease treated with subcutaneous apomorphine infusion or deep brain stimulation of the subthalamic nucleus. J Neurol Neurosurg Psychiatry. 2006; 77(4): 450-453.

11. Martinez-Martin P, Reddy P, Katzenschlager R, Antonini A, Todorova A, Odin P, et al. EuroInf: a multicenter comparative observational study of apomorphine and levodopa infusion in Parkinson's disease. Mov Disord. 2015; 30(4): 510-516.

12. Katzenschlager R, Poewe W, Rascol O, Trenkwalder C, Deuschl G, Chaudhuri R, et al. Double-blind, randomized, placebo-controlled, Phase III study (TOLEDO) to evaluate the efficacy of apomorphine subcutaneous infusion in reducing OFF time in Parkinson's disease patients with motor fluctuations not well controlled on optimized conventional treatment. Proceedings of the 21st International Congress of Parkinson's Disease and Movement Disorders; 2017 June 4-8; Vancouver, Canada. Mov Disord 2017; 32(Suppl 2): 518-519.
13. Kimber TE, Fang J, Huddy LJ, Thompson PD. Long-term adherence to apomorphine infusion in patients with Parkinson disease: a 10-year observational study. Intern Med J. 2017; 47(5): 570-573.

14. Borgemeester RW, Drent M, van Laar T. Motor and non-motor outcomes of continuous apomorphine infusion in 125 Parkinson's disease patients. Parkinsonism Relat Disord. 2016; 23: 17-22.

15. Tyne HL, Parsons J, Sinnott A, Fox SH, Fletcher NA, Steiger MJ. A 10 year retrospective audit of long-term apomorphine use in Parkinson's disease. J Neurol. 2004; 251(11): 1370-1374.

16. Defer GL, Widner H, Marié RM, Rémy P, Levivier M. Core assessment program for surgical interventional therapies in Parkinson's disease (CAPSIT-PD). Mov Disord. 1999; 14(4): 572-584.

17. Postuma RB, Berg D, Stern M, Poewe W, Olanow CW, Oertel W, et al. MDS clinical diagnostic criteria for Parkinson's disease. Mov Disord. 2015; 30(12): 1591-1601.

18. Goetz CG, Tilley BC, Shaftman SR, Stebbins GT, Fahn S, MartinezMartin P, et al. Movement Disorder Society-sponsored revision of the Unified Parkinson's Disease Rating Scale (MDS-UPDRS): scale presentation and clinimetric testing results. Mov Disord. 2008; 23(15): 2129-2170.

19. Guy W. Clinical Global Impressions (CGI) Scale, Modified. In: Rush AJ; Task Force for the Handbook of Psychiatric Measures, ed. Handbook of Psychiatric Measures. 1st ed. Washington, DC: American Psychiatric Association; 2000.

20. Trenkwalder C, Chaudhuri KR, García Ruiz PJ, LeWitt P, Katzenschlager R, Sixel-Döring F, et al. Expert Consensus Group report on the use of apomorphine in the treatment of Parkinson's disease - Clinical practice recommendations. Parkinsonism Relat Disord. 2015; 21(9): 1023-1030.

21. Fernández-Pajarín G, Sesar Á, Ares B, Castro A. Evaluating the Efficacy of Nocturnal Continuous Subcutaneous Apomorphine Infusion in Sleep Disorders in Advanced Parkinson's Disease: The APO-NIGHT Study. J Parkinsons Dis. 2016; 6(4): 787-792.

22. Antonini A, Tolosa E, Mizuno Y, Yamamoto M, Poewe WH. A reassessment of risks and benefits of dopamine agonists in Parkinson's disease. Lancet Neurol. 2009; 8(10): 929-937.

23. Gancher ST, Nutt JG, Woodward WR. Apomorphine infusional therapy in Parkinson's disease: clinical utility and lack of tolerance. Mov Disord. 1995; 10(1): 37-43.

24. Gancher S, Nutt J. Tolerance to apomorphine develops and reverses rapidly. Mov Disord. 2010; 25(6): 803-804.

25. Storch A, Schneider CB, Wolz M, Stürwald Y, Nebe A, Odin P, et al. Nonmotor fluctuations in Parkinson disease: severity and correlation with motor complications. Neurology. 2013; 80(9): 800-809.

26. Alegret M, Valldeoriola F, Martí M, Pilleri M, Junqué C, Rumià J, et al. Comparative cognitive effects of bilateral subthalamic stimulation and subcutaneous continuous infusion of apomorphine in Parkinson's disease. Mov Disord. 2004; 19(12):1463-1469.

27. Daley DJ, Myint PK, Gray RJ, Deane KH. Systematic review on factors associated with medication non-adherence in Parkinson's disease. Parkinsonism Relat Disord. 2012; 18(10): 1053-1061.

28. Chaudhuri KR, Qamar MA, Rajah T, Loehrer P, Sauerbier A, Odin P, et al. Non-oral dopaminergic therapies for Parkinson's disease: current treatments and the future. NPJ Parkinsons Dis. 2016; 2: 16023.

29. Todorova A, Chaudhuri KR. Subcutaneous apomorphine and nonmotor symptoms in Parkinson's disease. Parkinsonism Relat Disord. 2013; 19(12): 1073-1078.

30. Himeno E, Ohyagi Y, Ma L, Nakamura N, Miyoshi K, Sakae N, et al. Apomorphine treatment in Alzheimer mice promoting amyloid- $\beta$ degradation. Ann Neurol. 2011; 69(2): 248-256. 